\title{
Interrelation between the relative fat mass index and other obesity indices in predicting clinical severity and prognosis of acute myocardial infarction
}

\author{
Marko Mornar Jelavic ${ }^{1,2}$, Zdravko Babic ${ }^{3,4,5}$, Hrvoje Pintaric ${ }^{2,6}$ \\ ${ }^{1}$ Institute for Cardiovascular Prevention and Rehabilitation, Zagreb, Croatia \\ ${ }^{2}$ School of Dental Medicine, University of Zagreb, Zagreb, Croatia \\ ${ }^{3}$ School of Medicine, University of Zagreb, Zagreb, Croatia \\ ${ }^{4}$ Faculty of Kinesiology, University of Zagreb, Zagreb, Croatia \\ ${ }^{5}$ Coronary Care Unit, Department of Cardiology, Internal Medicine Clinic, Sestre Milosrdnice University Hospital Center, Zagreb, Croatia \\ ${ }^{6}$ Department of Emergency Medicine, Internal Medicine Clinic, Sestre Milosrdnice University Hospital Center, Zagreb, Croatia
}

Adv Interv Cardiol 2020; 16, 2 (60): 198-201

DOI: https://doi.org/10.5114/aic.2020.96064

\section{Introduction}

Central obesity is directly associated with insulin resistance, dyslipidemia and inflammation, which lead to atherosclerotic vascular disease [1]. There is a positive association of central obesity, as well as a negative association of overall obesity, with higher mortality in acute coronary syndrome, as body mass index (BMI) does not adequately discriminate the difference between body fat and lean muscle mass [2]. The newest obesity parameter, the relative fat mass index (RFMI), was more accurate for body fat-defined obesity and more accurate than BMI for those with a high body fat percentage [3].

\section{Aim}

We investigated the unknown interrelation between the RFMI and other obesity indices in predicting clinical severity and prognosis of acute ST-elevation myocardial infarction (STEMI) treated with primary percutaneous coronary intervention (PCI).

\section{Material and methods}

This prospective study, approved by the appropriate ethics committee, included 250 patients with acute STEMI treated with primary $\mathrm{PCl}$. The inclusion criteria were: presenting within $12 \mathrm{~h}$ from the onset of symptoms (history of chest pain/discomfort lasting for 10$20 \mathrm{~min}$ or more, not responding fully to nitroglycerine), persistent ST-segment elevation on electrocardiography (ECG) in at least two consecutive leads or (presumed) new left bundle branch block (LBBB), and elevated cardiac laboratory biomarkers (cardiac troponin T (cTnT) and creatine kinase (CK)). The diagnosis of acute STEMI was established and primary $\mathrm{PCl}$ performed using the European Society of Cardiology criteria [4, 5]. After primary $\mathrm{PCl}$, patients were classified into two groups (with/without RFMI obesity) which were analyzed by baseline, as well as severity and prognostic parameters of acute STEMI.

Baseline demographic and medical history parameters included gender, age, hypertension, dyslipidemia, hyperglycemia, anthropometry, smoking, known family history of cardiovascular events (MI, stroke), previous $\mathrm{MI}$, previous $\mathrm{PCl}$ and coronary artery bypass grafting (CABG). Anthropometric baseline data included BMI, waist circumference (WC), waist-to-hip (WHR) and waistto-height ratio (WHtR). RFMI was calculated using the equation RFMI $=64-(20 \times$ height/waist $)+(12 \times$ sex $)$, where sex $=0$ for men and 1 for women [3]. Increased RFMI values were defined as $\geq 25 \%, \geq 28 \%$ and $\geq 30 \%$ for males aged 20-39, 40-59 and 60-79 years, respectively. For females, increased RFMI values were defined as $\geq 39 \%, \geq 40 \%$ and $\geq 42 \%$ for ages $20-39,40-59$ and 60-79 years, respectively.

The severity of acute STEMI included: clinical presentation (angina pectoris, dyspnea, and length of hospital stay), in-hospital complications (arrhythmias, heart failure, cardiogenic shock, cardiac arrest, mechanical ventilation, reinfarction, repeated $\mathrm{PCl}$, mortality, and total in-hospital complications), coronary angiography, laboratory (creatinine clearance, maximal CTnT and CK) and

\section{Corresponding author:}

Marko Mornar Jelavic MD, PhD, Institute for Cardiovascular Prevention and Rehabilitation, Zagreb, Croatia, phone: +38 5(91)7826135,

e-mail: mjelavic@yahoo.com

Received: 13.12.2019, accepted: 30.03.2020. 
echocardiography findings (left ventricular ejection fraction - LVEF).

Coronary angiography was performed by applying a monoplane system (Axiom Artis, Siemens, Erlangen, Germany) [5]. Patients received $70 \mathrm{IE} / \mathrm{kg}$ of unfractionated heparin, $300 \mathrm{mg}$ of aspirin, a loading dose of $600 \mathrm{mg}$ of clopidogrel, and a GPIIb/IIla inhibitor according to the judgment of an interventional cardiologist. Stenosis of more than $50 \%$ was considered clinically significant. It was measured with the system software at all patients. We analyzed the number of significantly narrowed coronary arteries (CAs), their segments (proximal, middle and distal) [6], and the number, length and diameter of used stents.

Serum CK activity was measured by spectrophotometry (Olympus 680, Beckman Coulter Inc., California, USA), while cTnT levels were measured by electrochemiluminescence (ECL) assay (Cobas e411, Roche Diagnostics, Sussex, UK). During hospitalization, echocardiography was performed in all patients (Acuson Sequoia 512, Siemens, Munich, Germany) [7].

During hospitalization, 19 (7.6\%) patients died and 231 (92.4\%) entered 12-month prognostic evaluation with monitoring of major adverse cardiovascular events (MACE): cardiac (reinfarction, restenosis, new stenosis, urgent $C A B G$, other (e.g. heart failure)) and non-cardiac rehospitalizations (stroke, other (e.g. peripheral artery disease)), and mortality. Data were collected by medical examination, checking medical documentation, or telephone contact with patients, family members or home physicians.

\section{Statistical analysis}

Qualitative data were presented in absolute number and percentage. We used the $\chi^{2}$ test with Yates correction. Quantitative data were presented as median and range. Differences between the two groups were tested by Mann-Whitney $U$ test. Correlations between the anthropometric parameters with clinical severity and prognosis were investigated by Spearman's correlation and classified as very weak (0-0.19), weak (0.20-0.39), moderate $(0.40-0.59)$, strong $(0.60-0.79)$ and very strong (0.80-1.0). This guide also applies to negative correlations. We used Cox proportional-hazards regression for analyzing the effect of several risk factors on prognosis. The level of statistical significance was set at $p<0.05$ (Statistica 6.0 for Windows).

\section{Results}

RFMI obese subjects (55.2\%) had higher rates of arterial hypertension (80.4\% vs. $67.0 \%$ ) and dyslipidemia (81.2\% vs. $69.6 \%)$, higher median values of BMI (29.4 vs. $\left.25.6 \mathrm{~kg} / \mathrm{m}^{2}\right)$, WC (106 vs. $\left.93 \mathrm{~cm}\right)$, WHR (1.0 vs. 0.9$)$ and WHtR (0.62 vs. 0.53), higher rates of in-hospital complications (47.8\% vs. $33.9 \%)$, and higher median diameter of stents ( 3.5 vs. $3.0 \mathrm{~mm})(p<0.05)$, without significant differences between the two groups in other baseline parameters and parameters of clinical severity and prognosis.

We found a negative correlation of BMI with the significantly stenosed proximal CA segments and a positive correlation with stents diameter; positive correlations of WC with hospital stay and stents diameter; positive correlations of WHtR with hospital stay, in-hospital complications and stents diameter; a positive correlation of RFMI with in-hospital complications and negative correlations with parameters of myocardial necrosis (cTnT, CK) $(p<0.05)$ (Tables I and II).

After primary $\mathrm{PCl}$, all patients were taking dual antiaggregation therapy and statins, while the most commonly prescribed drugs were angiotensin converting enzyme inhibitors (ACEIs)/angiotensin-receptor blockers (ARBs) (72.1\%) and $\beta$-blockers (59.7\%), then diuretics (13.9\%) and calcium-channel blockers (CCB) (8.7\%). There were no significant correlations between the anthropometric parameters and MACE. However, there were significant positive correlations between the number of significantly stenosed CAs and MACE (rho $=0.24,95 \% \mathrm{Cl}$ : 0.12-0.36), and the number of proximal and distal significantly stenosed CA segments and MACE (rho $=0.14,95 \% \mathrm{Cl}$ : $0.01-$ 0.27 and rho $=0.19,95 \% \mathrm{Cl}: 0.06-0.31$, respectively); and a negative correlation between the LVEF and MACE ( $r$ ho = $-0.15,95 \% \mathrm{Cl}:-0.27$ to -0.02$)(p<0.05)$.

Cox analysis revealed no effect of several risk factors on MACE, i.e. of age (hazard ratio $(\mathrm{HR})=1.02$, 95\% Cl: 0.99 $1.05, p=0.060)$, male gender $(\mathrm{HR}=0.94,95 \% \mathrm{Cl}: 0.50-1.78$, $p=0.848)$, hypertension ( $\mathrm{HR}=1.57,95 \% \mathrm{Cl}: 0.77-3.23$, $p=0.203)$, dyslipidaemia $(\mathrm{HR}=0.82,95 \% \mathrm{Cl}: 0.44-1.55$, $p=0.555)$, hyperglycemia $(\mathrm{HR}=0.93,95 \% \mathrm{Cl}: 0.52-$ $1.66, p=0.801)$, smoking $(\mathrm{HR}=0.69,95 \% \mathrm{Cl}: 0.39-$ $1.23, p=0.208)$, in-hospital complications ( $\mathrm{HR}=0.98$, 95\% Cl: $0.55-1.77, p=0.949)$, creatinine clearance $(\mathrm{HR}=1.00,95 \% \mathrm{Cl}: 0.99-1.02, p=0.225), \mathrm{BMI}$ $(H R=0.97,95 \% \mathrm{Cl}: 0.91-1.04, p=0.974), W C(H R=1.00$, $95 \% \mathrm{Cl}: 0.98-1.02, p=0.999), \mathrm{WHR}(\mathrm{HR}=1.90,95 \% \mathrm{Cl}$ : $0.07-51.5, p=0.704), \mathrm{WHtR}(\mathrm{HR}=1.27,95 \% \mathrm{Cl}: 0.03-50.6$, $p=0.898)$ and RFMI $(\mathrm{HR}=1.00,95 \% \mathrm{Cl}: 0.97-1.05$, $p=0.745)$, except LVEF $(\mathrm{HR}=0.96,95 \% \mathrm{Cl}: 0.94-0.99$, $p=0.034)$ and significantly stenosed CAs (HR = 1.90, $95 \% \mathrm{Cl}: 1.35-2.62, p<0.001)$. After adjustment of MACE with these two factors, we found the effect of significantly stenosed CAs on MACE ( $\mathrm{HR}=1.78,95 \% \mathrm{Cl}: 1.26-2.52$, $p=0.001$.

\section{Discussion}

Studies have reported that overall obese patients have greater CA and stent diameters, normal LVEF, lower CK levels, lower in-hospital and overall mortalities, and lower rates of MACE during 12-month follow-up [8-10]. In this study, this overall "obesity paradox" could explain 
Table I. Correlation between BMI, WC and clinical severity and prognosis of acute STEMI

\begin{tabular}{lcccc} 
Parameter & BMI (rho) $(95 \%$ Cl) & P-value & WC (rho) (95\% Cl) & $P$-value \\
\hline Hospital stay [days] & $0.07(-0.06$ to 0.20$)$ & 0.271 & $0.14(0.02-0.27)$ & 0.025 \\
\hline Clinical presentation & $-0.06(-0.18$ to 0.06$)$ & 0.331 & $-0.00(-0.13$ to 0.12$)$ & 0.978 \\
\hline In-hospital complications & $0.00(-0.12$ to 0.12$)$ & 0.998 & $0.07(-0.05$ to 0.19$)$ & 0.265 \\
\hline Maximal cTnT [ng/ml] & $-0.11(-0.23$ to 0.19$)$ & 0.098 & $-0.04(-0.17$ to 0.08$)$ & 0.503 \\
\hline Maximal CK [U/l] & $-0.04(-0.17$ to 0.08$)$ & 0.515 & $-0.02(-0.15$ to 0.10$)$ & 0.744 \\
\hline LVEF (\%) & $0.01(-0.12$ to 0.14) & 0.840 & $0.04(-0.09$ to 0.17$)$ & 0.537 \\
\hline Proximal CA segments & $-0.15(-0.27$ to -0.02$)$ & 0.019 & $-0.04(-0.17$ to 0.08$)$ & 0.508 \\
\hline Distal CA segments & $-0.11(-0.24$ to 0.01$)$ & 0.073 & $-0.08(-0.20$ to 0.04$)$ & 0.205 \\
\hline Stents & $-0.07(-0.19$ to 0.06$)$ & 0.320 & $-0.12(-0.25$ to 0.01$)$ & 0.063 \\
\hline Diameter of stents [mm] & $0.24(0.12-0.36)$ & $<0.001$ & $0.15(0.03-0.28)$ & 0.019 \\
\hline Length of stents [mm] & $0.06(-0.07$ to 0.19$)$ & 0.381 & $0.12(-0.01$ to 0.24$)$ & 0.081 \\
\hline Total MACE & $-0.03(-0.16$ to 0.09$)$ & 0.624 & $-0.00(-0.13$ to 0.13$)$ & 0.996
\end{tabular}

BMI - body mass index, CAs - coronary arteries, Cl - confidence interval, CK - creatine kinase, CTNT - cardiac troponin T, LVEF - left ventricle ejection fraction, MACE - major adverse cardiovascular events, RFMI - relative fat mass index, STEMI - ST-elevation myocardial infarction, WC - waist circumference. Statistical significance defined as $p<0.05$.

Table II. Correlation between WHR, WHtR, RFMI and clinical severity and prognosis of acute STEMI

\begin{tabular}{|c|c|c|c|c|c|c|}
\hline Parameter & WHR (rho) $(95 \% \mathrm{Cl})$ & $P$-value & WHtR (rho) $(95 \% \mathrm{Cl})$ & $P$-value & RFMI (rho) $(95 \% \mathrm{Cl})$ & $P$-value \\
\hline Hospital stay [days] & $0.04(-0.09$ to 0.17$)$ & 0.543 & $0.14(0.02-0.26)$ & 0.028 & $0.12(-0.00$ to 0.25$)$ & 0.058 \\
\hline Clinical presentation & $-0.04(-0.17$ to 0.08$)$ & 0.495 & $-0.02(-0.15$ to 0.10$)$ & 0.716 & $0.04(-0.08$ to 0.17$)$ & 0.505 \\
\hline In-hospital complications & $-0.06(-0.18$ to 0.06$)$ & 0.346 & $0.12(0.00-0.24)$ & 0.049 & $0.16(0.04-0.28)$ & 0.010 \\
\hline Maximal cTnT [ng/ml] & $-0.03(-0.15$ to 0.10$)$ & 0.687 & $-0.10(-0.22$ to 0.03$)$ & 0.119 & $-0.18(-0.29$ to -0.06$)$ & 0.006 \\
\hline Maximal CK [U/I] & $-0.00(-0.13$ to 0.12$)$ & 0.977 & $-0.08(-0.20$ to 0.05$)$ & 0.210 & $-0.17(-0.28$ to -0.05$)$ & 0.010 \\
\hline LVEF (\%) & $0.07(-0.06$ to 0.20$)$ & 0.285 & $0.05(-0.08$ to 0.17$)$ & 0.469 & $0.06(-0.07$ to 0.19$)$ & 0.377 \\
\hline Proximal CA segments & $-0.00(-0.13$ to 0.12$)$ & 0.950 & $-0.00(-0.13$ to 0.12$)$ & 0.973 & $0.01(-0.12$ to 0.13$)$ & 0.925 \\
\hline Distal CA segments & $-0.08(-0.20$ to 0.05$)$ & 0.236 & $-0.05(-0.18$ to 0.07$)$ & 0.389 & $0.04(-0.08$ to 0.17$)$ & 0.492 \\
\hline Stents & $-0.04(-0.17$ to 0.09$)$ & 0.553 & $-0.09(-0.22$ to 0.04$)$ & 0.156 & $-0.12(-0.24$ to 0.01$)$ & 0.081 \\
\hline Diameter of stents [mm] & $0.08(-0.05$ to 0.21$)$ & 0.226 & $0.14(0.01-0.27)$ & 0.031 & $0.03(-0.10$ to 0.15$)$ & 0.696 \\
\hline Length of stents [mm] & $0.05(-0.08$ to 0.18$)$ & 0.447 & $0.08(-0.05$ to 0.21$)$ & 0.223 & $-0.01(-0.14$ to 0.12$)$ & 0.859 \\
\hline Total MACE & $0.03(-0.10$ to 0.16$)$ & 0.662 & $0.01(-0.12$ to 0.14$)$ & 0.936 & $0.04(-0.09$ to 0.16$)$ & 0.591 \\
\hline
\end{tabular}

CAs - coronary arteries, CK - creatine kinase, CTNT - cardiac troponin T, LVEF - left ventricle ejection fraction, MACE - major adverse cardiovascular events, RFMI relative fat mass index, STEMI - ST-elevation myocardial infarction, WHR - waist to hip ratio, WHtR - waist to height ratio. Statistical significance defined as $p<0.05$.

the lack of significant correlation between the values of $\mathrm{BMI}$ and clinical severity and prognosis, as well as the significant negative correlation of BMI with the number of significantly stenosed proximal CA segments.

Increased WC is associated with greater myocardial necrosis and worse LVEF in acute MI [11, 12]. We found the central "obesity paradox", with no significant correlation between the values of WC and clinical severity and prognosis, except a positive significant correlation of WC with hospital duration and stent diameter.
Patients with acute STEMI and increased WHR more frequently have heart failure; WHR is an independent predictor of 6-month mortality [13]. Our study revealed another example of the central "obesity paradox", with no significant correlation between the values of WHR and clinical severity and prognosis.

Among the obesity indices, WHtR has the strongest positive correlation with CAD [14]. Our values of WHtR positively correlated with hospital stay, in-hospital complications, and diameter of stents. 
Other authors reported that the RFMI was more accurate than BMI to estimate whole-body fat percentage and improved body fat-defined obesity misclassification among American adult individuals of Mexican, European or African ethnicity [3]. In this study, the values of RFMI positively correlated with in-hospital complications and negatively with laboratory parameters of myocardial necrosis (cTnT, CK).

Finally, the number of significantly stenosed CAs positively correlated with MACE, which is consistent with the literature data [15].

\section{Conclusions}

RFMI and WHtR are superior in predicting clinical severity (hospital stay, in-hospital complications) of acute STEMI, while none of the obesity indices have a role in predicting prognosis. We propose more frequent use of RFMI and WHtR in everyday clinical work in patients suffering from myocardial infarction.

\section{Conflict of interest}

The authors declare no conflict of interest.

\section{References}

1. Lee CD, Jacobs DR Jr, Schreiner PJ, et al. Abdominal obesity and coronary artery calcification in young adults: the Coronary Artery Risk Development in Young Adults (CARDIA) Study. Am J Clin Nutr 2007; 86: 48-54.

2. Coutinho T, Goel K, de Sá Corrêa D, et al. Central obesity and survival in subjects with coronary artery disease: a systematic review of the literature and collaborative analysis with individual subject data. J Am Coll Cardiol 2011; 57: 1877-86.

3. Woolcott OO, Bergman RN. Relative fat mass (RFM) as a new estimator of whole-body fat percentage - a cross-sectional study in American adult individuals. Sci Rep 2018; 8: 10980.

4. Ibanez B, James S, Agewall S, et al.; ESC Scientific Document Group. 2017 ESC Guidelines for the management of acute myocardial infarction in patients presenting with ST-segment elevation: the Task Force for the management of acute myocardial infarction in patients presenting with ST-segment elevation of the European Society of Cardiology (ESC). Eur Heart J 2018; 39: 119-77.

5. Windecker S, Kolh P, Alfonso F, et al. 2014 ESC/EACTS Guidelines on myocardial revascularization: The Task Force on Myocardial Revascularization of the European Society of Cardiology (ESC) and the European Association for Cardio-Thoracic Surgery (EACTS). Developed with the special contribution of the European Association of Percutaneous Cardiovascular Interventions (EAPCI). Eur Heart J 2014; 35: 2541-619.

6. Austen WG, Edwards JE, Frye RL, et al. A reporting system on patients evaluated for coronary artery disease. Report of the Ad Hoc Committee for Grading of Coronary Artery Disease, Council on Cardiovascular Surgery, American Heart Association. Circulation 1975; 51: 5-40.

7. Cheitlin MD, Armstrong WF, Aurigemma GP, et al.; American College of Cardiology; American Heart Association; American Society of Echocardiography. ACC/AHA/ASE 2003 guideline update for the clinical application of echocardiography: summary article: a report of the American College of Cardiology/ American Heart Association Task Force on Practice Guidelines (ACC/AHA/ASE Committee to Update the 1997 Guidelines for the Clinical Application of Echocardiography). Circulation 2003; 108: 1146-62.

8. Gurm HS, Brennan DM, Booth J, et al. Impact of body mass index on outcome after percutaneous coronary intervention (the obesity paradox). Am J Cardiol 2002; 90: 42-5.

9. Kosuge M, Kimura K, Kojima S, et al.; Japanese Acute Coronary Syndrome Study (JACSS) Investigators. Impact of body mass index on in-hospital outcomes after percutaneous coronary intervention for ST segment elevation acute myocardial infarction. Circ J 2008; 72: 521-5.

10. Kang WY, Jeong MH, Ahn YK, et al.; Korea Acute Myocardial Infarction Registry Investigators. Obesity paradox in Korean patients undergoing primary percutaneous coronary intervention in ST-segment elevation myocardial infarction. J Cardiol 2010; 55: 84-91.

11. Iglesias Bolaños P, Olivar Roldán J, Peñalver Talavera D, Díaz Guardiola P, Vega Piñero B, Monereo Megías S. [Effect of abdominal obesity on size of myocardial infarction]. Endocrinol Nutr 2009; 56: 4-8.

12. Azarfarin R, Samadikhah J, Shahvalizadeh R, Golzari SE. Evaluation of anthropometric indices of patients with left ventricle dysfunction fallowing first acute anterior myocardial infarction. J Cardiovasc Thorac Res 2012; 4: 11-5.

13. Lee SH, Park JS, Kim W, et al.; Korean Acute Myocardial Infarction Registry Investigators. Impact of body mass index and waistto-hip ratio on clinical outcomes in patients with ST-segment elevation acute myocardial infarction (from the Korean Acute Myocardial Infarction Registry). Am J Cardiol 2008; 102: 957-65.

14. Sabah KM, Chowdhury AW, Khan HI, et al. Body mass index and waist/height ratio for prediction of severity of coronary artery disease. BMC Res Notes 2014; 7: 246.

15. Sorajja P, Gersh BJ, Cox DA, et al. Impact of multivessel disease on reperfusion success and clinical outcomes in patients undergoing primary percutaneous coronary intervention for acute myocardial infarction. Eur Heart J 2007; 28: 1709-16. 